\title{
Enabling Relay-aided IP Communications in 802.11p/WAVE Networks
}

\author{
Sandra Céspedes*† and Xuemin (Sherman) Shen* \\ * Department of Electrical and Computer Engineering, University of Waterloo, Waterloo, Canada \\ $\dagger$ Department of Information and Communications Technology, Icesi University, Cali, Colombia \\ \{slcesped,xshen\}@bbcr.uwaterloo.ca
}

\begin{abstract}
Vehicular communications networks and the 802.11p/WAVE technology have become a fundamental platform for providing real-time access to safety and entertainment information in vehicular scenarios. In particular, IP-based infotainment applications are key to leverage the deployment costs of the 802.11p/WAVE network. However, the operation of IP in the standard 802.11p/WAVE and its throughput performance are still unclear, as the standard recommendations for the operation of IPv6 over WAVE are rather minimal. Consequently, this paper focuses on the provision of infrastructure-based IP communications in $802.11 \mathrm{p} / \mathrm{WAVE}$ networks, and proposes the Vehicular IP in WAVE (VIP-WAVE) framework, which defines a new per-user assignment and mobility management of IPv6 addresses supported by Proxy Mobile IPv6 over WAVE, and exploits relay-aided communications to improve the network performance along roads with different levels of infrastructure presence. Extensive simulations are carried out to demonstrate the effectiveness of the proposed framework to enhance the performance of IP applications in the vehicular network.
\end{abstract}

\section{INTRODUCTION}

The $802.11 \mathrm{p} /$ WAVE standards propose a set of technologies and protocols to establish vehicle-to-vehicle (V2V), and vehicle-to-infrastructure (V2I/I2V) communications. Although important research efforts have been devoted to guarantee the delivery of safety information in vehicular networks, the support of infotainment traffic has been traditionally displaced as a problem to be solved by other types of access networks, such as $3 \mathrm{G}$ or WiFi. Nevertheless, the deployment of infotainment applications, and consequently of IP-based communications, would certainly help accelerate the market penetration and leverage the deployment costs of the 802.11p/WAVE network.

As a result, the $802.11 \mathrm{p} /$ WAVE standard has also included IPv6 support in its stack of protocols. However, the recommendations for the operation of IPv6 over WAVE are rather minimal [1]. Moreover, the protocols in which the operation of IPv6 relies for addressing configuration and IP-to-link-layer address translation (e.g., the Neighbor Discovery protocol) are not recommended in the standard WAVE [2].

Consequently, we have identified three major limitations in the current $802.11 \mathrm{p} / \mathrm{WAVE}$ standard for the support of IPbased communications: 1) a lack of a duplicate address detection mechanism; 2) a lack of seamless communications for extended IP services (i.e., services that are continuously provided throughout the entire network); and 3) a lack of support

(C) 2012 IEEE. Personal use of this material is permitted. Permission from IEEE must be obtained for all other uses, in any current or future media, including reprinting/republishing this material for advertising or promotional purposes, creating new collective works, for resale or redistribution to servers or lists, or reuse of any copyrighted component of this work in other works.

Published in Proc. Globecom'12. DOI:10.1109/GLOCOM.2012.6504007 for multi-hop communications. With these open operational aspects of IPv6, providing access to IP-based applications, such as assisted parking, route management, and eventually Internet access, becomes a challenging task in $802.11 \mathrm{p} / \mathrm{WAVE}$ networks.

Although there have been previous works to evaluate the performance of infrastructure-based IP applications in vehicular environments, they employ the traditional $802.11 \mathrm{~b} / \mathrm{g}$ technology that does not resemble the intricacies defined in the $802.11 \mathrm{p} /$ WAVE standards for multi-channel access and IPbased communications. In [1], the limitations of the operation of IPv6 in WAVE have been identified, but their scope is to provide guidelines regarding the incompatibilities of the two technologies.

Therefore, this paper addresses the problem of infrastructure-based IP communications in 802.11p/WAVE networks, and proposes the Vehicular IP in WAVE (VIPWAVE): a framework that defines an efficient mechanism for the assignment of unique IPv6 addresses to On Board Units (OBUs), and the support of IP mobility for extended IP services. The latter is based on the Proxy Mobile IPv6 protocol and boosted by relay-aided communications. Extensive simulations are employed to demonstrate that the performance of VIP-WAVE is superior to the proposed WAVE standard. We also demonstrate the benefits that relay-aided communications may bring for a better support of IP services in $802.11 \mathrm{p} /$ WAVE networks, specially in adversary conditions, where Road Sise Units (RSUs) are scarcely distributed along the road segments.

The remainder of this paper is organized as follows. Section II describes how IP services are supported in the standard WAVE and reviews the related work. Sections III presents our network model. Sections IV and V introduce the architecture of the proposed framework and its extensions for relay-aided communications. The performance evaluation of VIP-WAVE is presented in section VI, and the concluding remarks are provided in section VII.

\section{RELATED WORK}

\section{A. The standard $802.11 \mathrm{p} / \mathrm{WAVE}$}

The standards for Wireless Access in Vehicular Environments (WAVE) define the medium-access channel capabilities for multi-channel operation, and the management and data delivery services between WAVE devices [2]. WAVE frequency spectrum is divided into 1 control channel $(\mathrm{CCH})$, for safety and control information, and 6 service channels ( $\mathrm{SCH})$, for data services, each of $10 \mathrm{MHz}$ bandwidth. In addition, each channel has an independent instance of the CSMA/CA protocol for accessing the wireless media. If the OBU has a 
single $802.11 \mathrm{p}$ radio (i.e., single-PHY), then it continuously switches between $\mathrm{CCH}$ and SCHs for exchanging control and data packets. On the other hand, a multi-PHY OBU has more than one radio, hence, it is able to monitor the $\mathrm{CCH}$ and at the same time it can exchange data in one or more SCHs.

In [2], the standard specifies the mandatory support of IPv6 link-local, global, and multicast addresses in WAVE devices. It also states that "WAVE devices may implement any Internet Engineering Task Force (IETF) protocol"; however, it does not specify the operation conditions for the Neighbor Discovery (ND) protocol. IP services are broadcast by RSUs in Wave Service Advertisement (WSA) messages, which include ServiceInfo segments, Channelinfo segments, and up to one WaveRoutingAdvertisement (WRA) segment. A ServiceInfo includes information such as the IP address of the hosting server, and the minimum received power level recommended to accept the service (RCPI threshold). A Channelinfo includes the transmission power, data rate, and channel number in which a service is provided. The WRA includes the IP prefix that OBUs should employ to access the IP services announced in the WSA.

From the described operation of IP services in 802.11p/WAVE networks, one can identify the following limitations:

Lack of duplicate address detection mechanism. A WAVE user interested in a specific IP service is allocated with the same IP prefix of all other users subscribing to any IP service announced in the same WSA. Consequently, OBUs should perform a duplicate address detection (DAD) procedure, to guarantee the uniqueness of IP addresses among all users, especially because the WAVE standard allows for MAC readdressing to achieve pseudonymity. Nonetheless, the Neighbor Discovery protocol, which includes the standard DAD procedure for IPv6, is not recommended in WAVE. Furthermore, in the case the user is consuming an extended service (i.e., a service announce by all RSUs), even if it has initially performed $\mathrm{DAD}$, the procedure will be invalidated as soon as the vehicle moves to a new service area, since the set of neighbors will also change.

Lack of seamless communications for extended services. Suppose the DAD problem is alleviated by having each RSU to advertise a unique set of IP prefixes among all the other RSUs. If an extended IP service is to be provided, this solution would cause breakage of service continuity, due to the OBU receiving different IP configuration information when roaming through different RSUs. Therefore, transport layer sessions would have to be reset in order to continue the communications.

Lack of relay-aided communications. The current standard allows for a WAVE user to consume infrastructure-based IP services only when there is a direct connection between RSU (i.e., WAVE provider) and user OBU. We argue this represents a limitation of the 802.11p/WAVE standards. Vehicular networks experience highly variable channel conditions due to mobility, obstacles, and interference. Therefore, it is desirable to take advantage of intermediate WAVE devices to relay packets from/to the infrastructure. This could not only improve the network capacity, but it could also represent a benefit for the relay OBU (e.g., the earning of credits for future rewards from the network [3]).

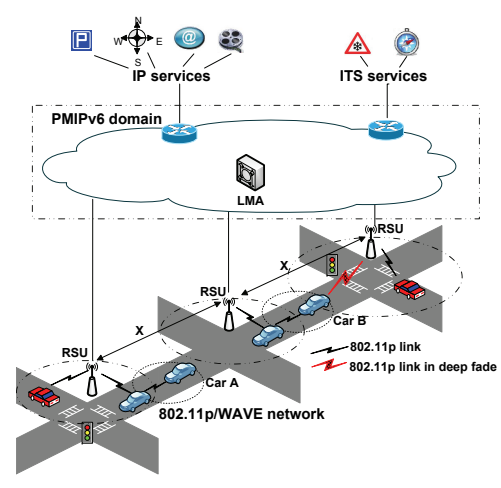

Fig. 1. IP-enabled 802.11p/WAVE network model

\section{B. Related Work}

IP becomes a natural solution for providing addressing services in WAVE, and for enabling the access to existent IP networks (e.g., the Internet), to legacy applications, and to innovative services. Therefore, IP addressing configuration in vehicular networks has been investigated in numerous studies [4]-[6]. While these studies enable IP configuration in moving vehicles, they are often limited to guarantee uniqueness in a specific area (e.g., around the leading vehicle acting as DHCP server [4], around the service area of RSU [5], or around a specific lane [6]), which may represent a limitation for the deployment of extended IP services and seamless communications in $802.11 \mathrm{p} / \mathrm{WAVE}$.

In terms of mobility management, host mobility solutions in vehicular networks that employ the Network Mobility Basic Support (NEMO) protocol are proposed and evaluated in [7][9]. Baldessari et al. [7] define a MANET-centric solution that exploits multi-hop communications, so that each vehicle is treated as a NEMO Mobile Router. Prakash et al. [8] propose a vehicle-assisted cross-layer handover scheme for vehicles to help relay signalling and data packets of a handover vehicle. Different from the aforementioned works, network-based mobility with Proxy Mobile IPv6 has been proposed in [10], for providing broadband Internet access in a transparent way in automotive scenarios.

In general, those schemes reduce the handover delay and improve the throughput in vehicular networks. However, none of them specifically consider the use of $802.11 \mathrm{p}$ for V2I communications. Instead, they employ a general 802.11 network for connectivity to the infrastructure, or theoretical performance evaluations. Thus, in our work, we propose a customized mobility management mechanism tailored to the characteristics of $802.11 \mathrm{p} /$ WAVE networks.

\section{NETWORK MODEL}

Consider the infrastructure-based vehicular network shown in Fig. 1. The connection to the infrastructure is provided by RSUs located along the road. Vehicles are equipped with OBUs that enable connections to the infrastructure and to other vehicles. RSUs and OBUs are equipped with 802.11p/WAVE radios, and are assumed to be multi-PHY. This way, we alleviate problems such as bandwidth wastage, longer queuing, and higher end-to-end delay, which have been previously identified as the consequences of the channel switching operation performed by $802.11 \mathrm{p}$ single-PHY radios [11]. 
We concentrate on the support of extended IP services in the $802.11 \mathrm{p} /$ WAVE network. Examples of extended IP services are mapping applications, route planning, and Internet access. In terms of connectivity, depending on the channel conditions, vehicles may establish direct connections to the RSU. However, there are times when vehicles are located in areas uncovered by the infrastructure (see car A in Fig. 1), or with a communication link in deep fade toward the RSU (see car B in Fig. 1). Inside such areas, we exploit the use of relay-aided communications.

We have selected the standard Proxy Mobile IPv6 (PMIP) protocol [10] to manage the IP mobility of the OBUs. PMIP defines two entities: 1) the Mobility Anchor Gateway (MAG), in charge of detecting when a node joins or moves in the PMIP domain; it also emulates a home link for the mobile node; and 2) the Local Mobility Anchor (LMA), which is the central entity in charge of assigning the IP prefixes to mobile nodes. The MAG and LMA use Proxy Binding Updates (PBU) and Proxy Binding Acknowledgments (PBA), respectively, for requesting and assigning IP prefixes.

The general integration of PMIP with the $802.11 \mathrm{p} / \mathrm{WAVE}$ network has been illustrated in Fig. 1. We consider the whole 802.11p/WAVE network as a single PMIP domain, and colocate the MAG functionalities with the RSU.

\section{VIP-WAVE ARCHITECTURE}

As denoted in section II-A, the current standard defines the announcement of a per-WSA IP prefix, which forces WAVE users of all IP services announced in a specific WSA (up to 32 services per WSA) to belong to the same IP network. This causes not only the necessity to frequently detect duplicate addresses when moving across different RSUs, but also contradicts one of the main requirements IPv6 has for the link-layer model, which indicates that all nodes belonging to the same IP prefix should be able to communicate directly with each other. Such requirement is not met when nodes may be scattered throughout different SCHs and may eventually also move away from each other.

The general idea behind our framework is to address that limitation by integrating IP configuration and IP mobility in order to provide continuity for extended IP services.

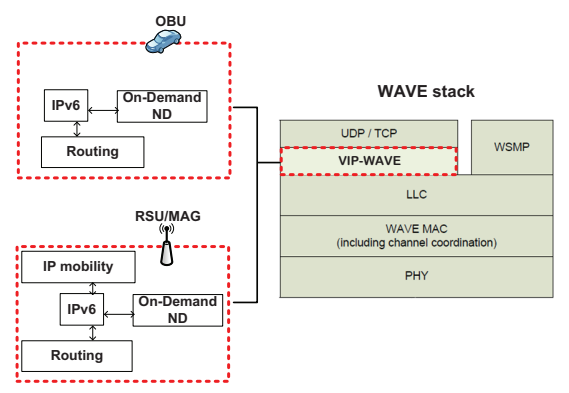

Fig. 2. Vehicular IP in WAVE (VIP-WAVE) architecture

We intend to enable a per-OBU IP prefix for the access to extended IP services and for guaranteeing seamless communications. Moreover, we intend to extend the coverage of IP services by extending the access to users located two hops away from the RSU, i.e., by exploiting relay-aided communications.
The architecture of VIP-WAVE is illustrated in Fig. 2. VIPWAVE is located in the data plane of the WAVE stack of protocols and it defines three main components that interact with the standard IPv6: 1) the IP addressing and mobility block; 2) the on-demand Neighbor Discovery block, which is a light-weight adaptation of the standard ND; and 3) the routing block, which enables relay selection for relay-aided communications.

In the following sections, we first describe the interaction of VIP-WAVE's components for the support of IP services to vehicles directly connected (i.e., 1-hop connection) to the infrastructure, and then we introduce the extensions required for enabling support of relay-aided communications.

\section{A. IP service establishment}

When an RSU announces an IP service, it includes the global IP address of the hosting server, its own MAC address that identifies it as the WAVE provider, and the RCPI threshold (i.e., the minimum received power level recommended) in a ServiceInfo segment. That segment is later transmitted in a WSA over the $\mathrm{CCH}$, as specified in [2]. Since OBUs have a radio dedicated to monitor the $\mathrm{CCH}$, all 1-hop users in the area of coverage of the RSU may receive the WSA. Upon WSA reception, an OBU interested in consuming the IP service proceeds in the following way:

The OBU tunes a radio to the $\mathrm{SCH}$ specified in the Channelinfo segment. At that point, the OBU does not have a global IP address to initiate communications with the hosting server; therefore, the IPv6 module requests the ondemand ND module to trigger a Router Solicitation (RS) message. The RS message is destined to the all-routers multicast address as indicated in the standard ND, and is handed to the routing module after determining the layer 2 next-hop address. Since the user is directly connected to the RSU, the routing module selects the WAVE provider MAC address (i.e., RSU MAC address) as the MAC-layer frame destination; thus, instead of multicast, the RS is delivered as a unicast message. The RSU then exchanges PBU/PBA messages with the LMA for IP prefix assignment, after which the RSU sends a unicast Router Advertisement (RA) message to the OBU. The RA message includes all the information required by IPv6 for a proper configuration.

Once the global IP address has been configured, the OBU may start exchanging IP data packets with the hosting server. Note that no DAD mechanism is required after IP address configuration, since the IP address uniqueness is guaranteed by having an IP prefix uniquely assigned per-OBU.

\section{B. Handover of IP services}

An OBU transitions through the service area of different RSUs at vehicular speeds. Therefore, we introduce a handover mechanism that allows for seamless communications of extended IP services in the 802.11p/WAVE network. When an OBU is consuming an extended service, it continues monitoring the $\mathrm{CCH}$ while is roaming toward a new RSU. Consequently, upon the OBU's reception of a WSA that announces the same extended service but from a different WAVE provider (i.e., the WAVE provider field in ServiceInfo now includes a different MAC address) serves as a movement detection hint that is notified by the MAC layer to VIP-WAVE. Upon the movement notification, the on-demand ND module 
TABLE I

RELAY SETUP PROCEDURE IN VIP-WAVE

Procedure at user OBU
Relay detection
1: if (WSAs from the RSU are no longer received or received WSA signal $<$
RCPI threshold)
2: create a Service Info to announce the Relay Service solicitation.
3: include this OBU's ID and IP address in the extension fields of Service Info.
4: associate a Channel Info segment with SCH number of active IP service.
5: send a WSA with the Relay Service announcement in CCH.
6: else
7: keep using one-hop connection to RSU.
Relay setup
25: if (Relay Service announcement has been sent and received WSA with
26: Relay Maintenance announcement)
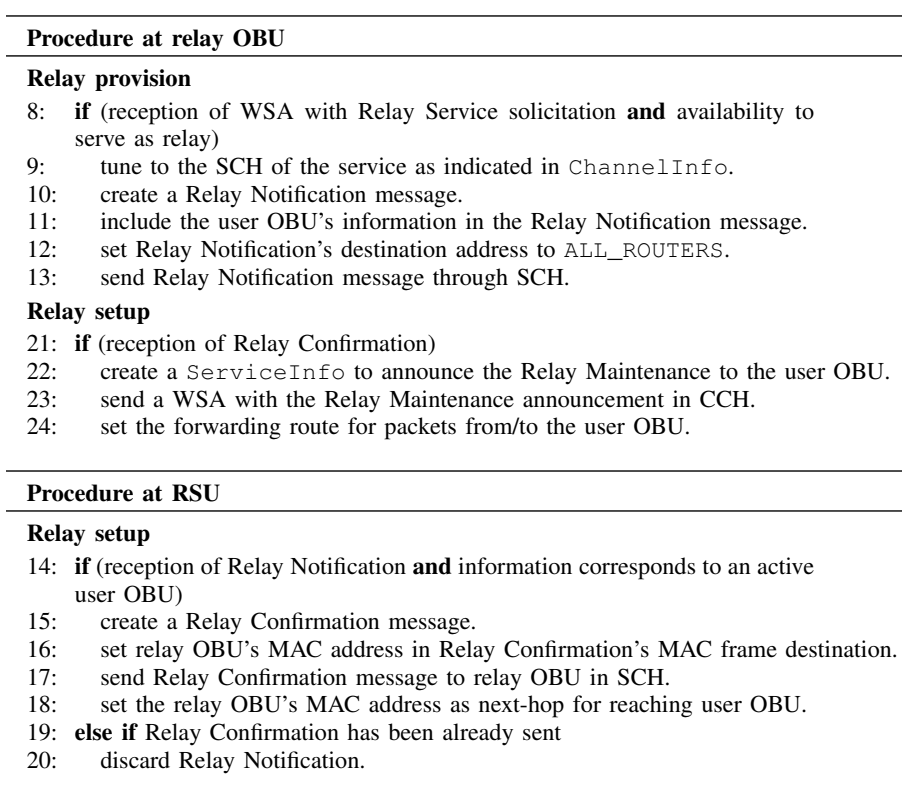

triggers the sending of an RS message, which is transmitted over the $\mathrm{SCH}$ in which the service is being provided.

The reception of the RS message is then employed by the new RSU for connection detection, so that it proceeds to exchange PBU/PBA signalling with the LMA. The new RSU sends an RA to the recently detected OBU as a response to the RS message. As a result, the LMA is able to resume packets forwarding toward the OBU as soon as it sends the PBA to the new RSU. The OBU, on the other hand, is able to resume packets transmission toward the hosting server once it receives the RA.

Note that our on-demand ND does not require the frequent sending of messages. We have replaced the necessity of receiving frequent RA messages by the reception of WSAs that are already defined in the standard. Thus, an IP prefix does not expire, unless announcements for the service that is currently being consumed are no longer received. In this way, the WSA message reception aids the VIP-WAVE layer in two ways: 1) it helps the maintenance of IP addresses by replacing the non-solicited RA messages; and 2) it solves the IP-to-linklayer address translation, because the WSA already includes the MAC address of the current WAVE provider. In addition, we alleviate possible congestion in the $\mathrm{CCH}$ by having the on-demand ND messages (e.g., RS or RA) being transmitted only over the SCH.

\section{VIP-WAVE EXTENSIONS FOR RELAY-AIDED COMMUNICATIONS}

In this section, we define the necessary features and services to extend the support of VIP-WAVE in 2-hop scenarios. We start by defining two services that are closely related: 1) the Relay Service, which is announced only when a user OBU requires another OBU to serve as a relay. A request for relay service may only be sent after the user OBU has started consuming a given service (i.e., after the OBU has acquired its IP configuration from the RSU); and 2) the Relay Maintenance, which is announced by the intermediate OBU that has been selected as a relay.

Since neighboring OBUs may also be consuming an IP service, only those OBUs with availability to serve as temporary relays will take action when they receive a Relay Service request. The procedure for setting up a relay OBU is located in the routing module and described in detail as follows.

\section{A. Routing through a relay}

Table I presents the algorithm for setting up communications through a relay. Once the procedure has been completed in all the involved parties, the RSU and user OBU have the necessary information for delivering packets through a 2-hop path, so that the exchange of IP packets may be resumed. Depending on the direction of traffic, the routing module operates in the following way for relay-aided communications:

1) Traffic from hosting server to user $O B U$ : Once the packet arrives at the RSU, the IP module queries the routing module about the next-hop to reach the user OBU. The routing module selects the relay OBU MAC address as the MAC-layer frame destination, since this has been configured by the relay setup procedure. The packet is then forwarded to relay OBU.

2) Traffic from user $O B U$ to hosting server: Once the data packet is generated at the user OBU, the IP module determines that the hosting server belongs to an external network; thus, it decides to send the packet toward the default gateway, which in this case is the RSU. The IP module then queries the routing module about the next-hop to reach the RSU. As configured by the relay setup procedure, the route to reach the RSU indicates the relay OBU as the next-hop; therefore, the relay OBU MAC address is selected as the MAC-layer frame destination. The packet is then forwarded to relay OBU.

If at any moment during the 2-hop communications, the user OBU detects again the reception of WSA directly from the RSU in the $\mathrm{CCH}$, and with a signal level above the RCPI threshold, then the user OBU sends a Router Solicitation to re-establish direct communications with the RSU. The Router Advertisement response message sent by the RSU is overheard and employed by the relay OBU for terminating the relay service.

\section{B. Handover in 2-hop scenarios}

When the vehicle is in motion, it may experience handovers of communications in different scenarios: 1) it may move the connection to a relay OBU, where both relay and user OBU remain in the service area of the same RSU; and 2) it may move the connection to a relay OBU, where the relay OBU is connected to a new RSU (i.e., different from the user OBU's current RSU). Note that the handover procedure when the vehicle maintains a direct connection to the RSU has been already defined in section IV-B. 
1) Handover to a relay in the same service area: In this scenario, the signalling required to maintain seamless communications is no different from that described in Table I. Since both relay OBU and user OBU remain in the service area of the same RSU, when the current RSU receives the Relay Notification message (step 14), it finds the information about the user OBU registered in the binding list of active IP users. Therefore, it does not require to trigger any signalling for IP mobility.

2) Handover to a relay in a different service area: The procedure for 2-hop handover to a different service area is illustrated in Fig. 3. In this scenario, the handover may be triggered by the conditions described in Table I (step 1), so the relay detection procedure is started. However, given that the relay is connected to a different service area, when the new RSU receives the Relay Notification message (step 14), it does not have an active tunnel configured for the user OBU. Therefore, the new RSU uses the Relay Notification message as a hint for connection detection, and triggers the PBU/PBA signalling toward the LMA. Once the PBA is received by the new RSU, it continues with the sending of Relay Confirmation (step 15) to the relay OBU. This message serves for triggering the Relay Maintenance announcements from relay OBU to user OBU (step 23), after which bi-directional communications are resumed.

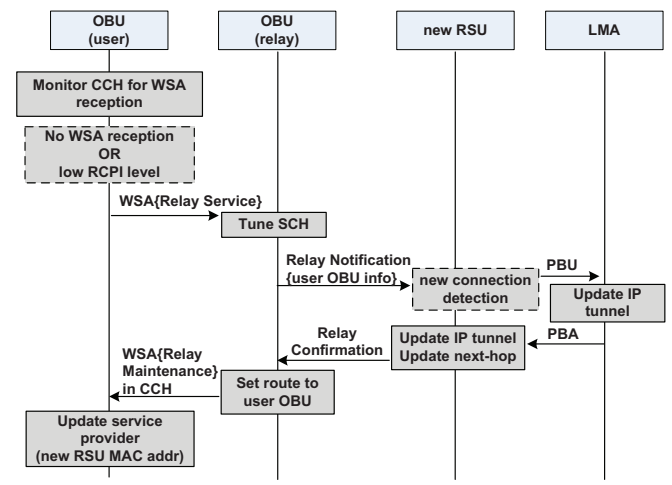

Fig. 3. Handover of extended IP services through a relay in VIP-WAVE

\section{Vi. Performance eValuation}

We have conducted simulations based on the discrete event simulator OMNeT++. RSUs and OBUs are equipped with two wireless interfaces transmitting in different channels around the $5.9 \mathrm{GHz}$ band. In this way, we emulate the multi-PHY capabilities with simultaneous transmissions over the $\mathrm{CCH}$ and the $\mathrm{SCH}$. Transmission power is set to $50 \mathrm{~mW}$ and $11 \mathrm{~mW}$ (i.e., $500 \mathrm{~m}$ and $250 \mathrm{~m}$ approximate radio range), for RSUs and OBUs, respectively, and with $6 \mathrm{Mbps}$ transmission rate. Each radio implements the Inetmanet 802.11p PHY and MAC model. Connectivity among nodes is initially determined by a unit disk model. However, signals are attenuated following a Log-normal propagation model with path-loss exponent of 2.4. We have also modified the Inetmanet package so that it delivers the OBU's received power to the network layer; in this way, we can employ not only the distance but also the RCPI threshold to determine connectivity between OBU and RSU. An Internet-located application server for the downloading of data traffic is connected to the 802.11p/WAVE network with an RTT of $40 \mathrm{~ms}$.
RSUs are uniformly distributed along the road segment, and the number of vehicles is given by the road density $\rho$ (vehicles per meter). Vehicles are randomly set as available relays according to the parameter $p_{r}$, which may vary from $40 \%$ to $100 \%$ of the population around the observed OBU. For those that are not available relays, they are transmitting in saturated mode with a packet size of $1 \mathrm{~KB}$. By default, the server transmission rate $\lambda_{d}$ is $100 \mathrm{Kbps}$ and the RCPI threshold has been set to $-85 \mathrm{dBm}$. In each experiment, we run randomly generated topologies and plot the results with the $95 \%$ confidence interval. The simulated IP session is active for $600 \mathrm{~s}$.

We compare our VIP-WAVE framework with the standard WAVE with no mobility management (WAVE-A), and with the standard WAVE with Proxy Mobile IPv6 (WAVE-B). In WAVE-A, each RSU announces a different prefix in the WRA segment, which forces vehicles to reset communications for extended IP services every time they roam to a different RSU. In WAVE-B, we assume the network supports Proxy Mobile IPV6. Although the standard does not indicates the protocol to be used for IP mobility, this scenario allows us to provide a fair comparison in terms of signalling delay and overhead between the standard and the proposed schemes.

We employ the nodal downstream throughput $T$, defined as the average rate of packets received at the user OBU, for the metric of comparison among the different schemes. Fig. 4(a) shows the throughput obtained when the distance $X$ between two consecutive RSUs increases from $250 \mathrm{~m}$ to $2000 \mathrm{~m}$. As shown in the figure, the performance of VIP-WAVE outperforms the standard one even when the same IP mobility protocol is employed. It is also observed how the effective throughput drops for all, as soon as $X>2 R$, where $R$ is the RSU radio range. This is due to the existence of uncovered areas between consecutive RSUs; in the case of VIP-WAVE, the greater $X$ is, the more the vehicle depends on the density for being able to find a 2-hop connection toward an RSU.

The impact of the vehicles velocity is illustrated in Fig. 4(b). Both VIP-WAVE and standard WAVE demonstrate to be stable for different average speeds. However, the extended area of coverage provided by the relay-aided communications in VIP-WAVE shows its benefit: it improves the effective throughput by nearly $20 \%$ compared to the standard WAVE. In order to evaluate the impact of the available number of OBUs willing to serve as relays, we test VIP-WAVE for different $p_{r}$. The results of these experiments are depicted in Fig. 4(c). It shows that even for a low availability of $40 \%$, the difference in the effective throughput is minimum. That means VIP-WAVE only requires one neighboring OBU to be available (and connected to the RSU) to take advantage of 2-hop connections in uncovered areas.

Fig. 5 illustrates the instantaneous throughput achieved by a randomly chosen vehicle during the simulated IP session. It shows the times at which 1-hop and 2-hop handovers occur, which helps understand the behavior during relayaided communications. Although the relayed connections only last for a few tens of seconds every time, the throughput improvement is high, in part because during 2-hop to 1-hop transitions the user OBU is able to maintain the reception of data packets while it re-establishes a bi-directional connection toward the RSU. It is also observed that the overhead incurred in establishing the relayed connection plays a minor impact in 


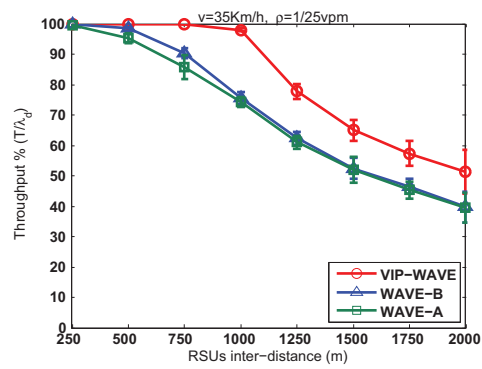

(a) Different RSUs inter-distances

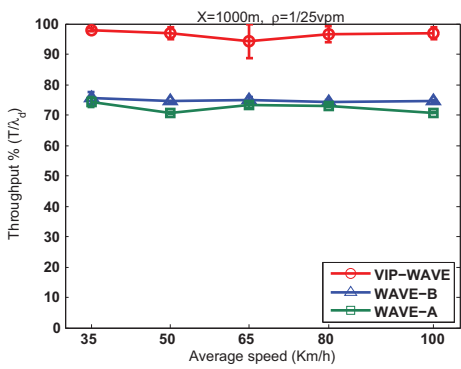

(b) Different average speeds

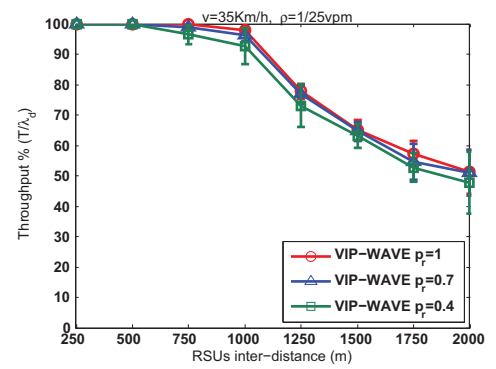

(c) Different relays availability

Fig. 4. Comparison of nodal download throughput

the overall performance of the end-to-end communications. Thus, the throughput remains fairly stable, and the relaying of packets helps reduce the total packet losses.

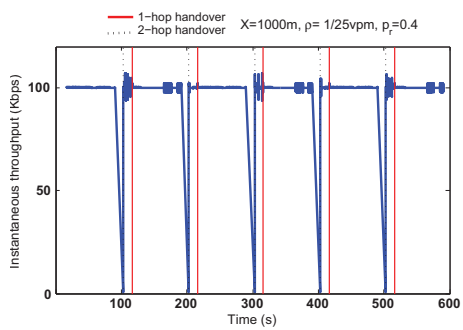

Fig. 5. Instantaneous throughput of relay-aided VIP-WAVE

Furthermore, as many IP applications are delay-sensitive, we evaluate the effect of two-hop communications in the data packets end-to-end delay. Fig. 6 depicts the latency experienced by individual packets received at the OBU during a session time. For those packets being transmitted through a two-hop connection in the 802.11p network, they perceive a slightly higher latency than those using a one-hop connection. However, the total delay, which is less than $37 \mathrm{~ms}$ in all cases, fits well into the delay requirements for the main multimedia applications, such as $150 \mathrm{~ms}$ for real time audio, and $250 \mathrm{~ms}$ for video conferencing and video streaming.

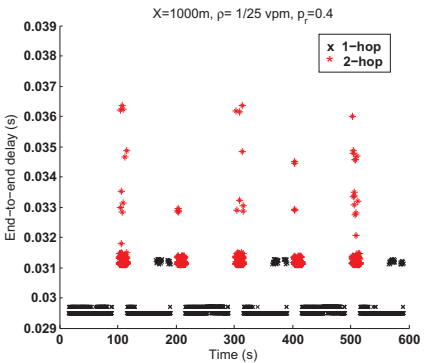

Fig. 6. Data packet end-to-end delay in VIP-WAVE

\section{CONCLUSION}

In this paper, we have proposed VIP-WAVE: a framework for the support of extended IP services in 801.11p/WAVE networks. VIP-WAVE is intended to overcome the limitations existent in the standard WAVE for the support of IP-based communications. It exploits network-based mobility and relayaided communications in order to provide smooth and seamless access to IP services in the vehicular network. By means of VIP-WAVE, we have achieved uniqueness of IP addressing assignment, seamless communications, and an extended service area of the RSU. Through extensive simulations, we have shown that a greater throughput is obtained by VIPWAVE, compared with the standard WAVE, even for highly scattered placement of RSUs along the road segments. For our future work, we will extend our framework to provide a more intelligent selection of relays that considers link duration and a relay rewarding scheme.

\section{REFERENCES}

[1] E. Baccelli, T. Clausen, and R. Wakikawa, "IPv6 Operation for WAVE Wireless Access in Vehicular Environments," in Proc. IEEE VNC, 2010 pp. $160-165$

[2] "1609.3-2010 IEEE Standard for Wireless Access in Vehicular Environments (WAVE) - Networking Services," IEEE Std 1609.3-2010 (Revision of IEEE Std 1609.3-2007), pp. 1-144, 2010.

[3] M. E. Mahmoud and X. Shen, "PIS: A Practical Incentive System for Multihop Wireless Networks," IEEE Trans. Veh. Technol., vol. 59, no. 8, pp. 4012-4025, Oct. 2010.

[4] S. D. Maria Fazio, "Automatic IP Address Configuration in VANETs," in Proc. ACM VANET, 2006, pp. 100-101.

[5] R. Baldessari, C. J. Bernardos, and M. Calderon, "GeoSAC - Scalable Address Autoconfiguration for VANET Using Geographic Networking Concepts," in Proc. IEEE PIMRC, 2008, pp. 1-7.

[6] T. Kato, K. Kadowaki, T. Koita, and K. Sato, "Routing and Address Assignment Using Lane/Position Information in a Vehicular Ad Hoc Network," in Proc. IEEE APSCC, 2008, pp. 1600-1605.

[7] R. Baldessari, W. Zhang, A. Festag, and L. Le, "A MANET-centric Solution for the Application of NEMO in VANET Using Geographic Routing," in Proc. TridentCom, 2008, pp. 1-7.

[8] A. Prakash, S. Tripathi, R. Verma, N. Tyagi, R. Tripathi, and K. Naik, "Vehicle Assisted Cross-Layer Handover Scheme in NEMO-based VANETs (VANEMO)," Int. J. of Internet Protocol Technology, vol. 6, no. $1 / 2$, p. 83 , Jun. 2011.

[9] S. Céspedes, X. Shen, and C. Lazo, "IP Mobility Management for Vehicular Communication Networks: Challenges and Solutions," IEEE Commun. Mag., vol. 49, no. 5, pp. 187-194, May 2011.

[10] I. Soto, C. J. Bernardos, M. Calderon, A. Banchs, and A. Azcorra, "Nemo-enabled Localized Mobility Support for Internet Access in Automotive Scenarios," IEEE Commun. Mag., vol. 47, no. 5, pp. 152159, May 2009.

[11] Y. Du, L. Zhang, Y. Feng, Z. Ren, and Z. Wang, "Performance Analysis and Enhancement of IEEE $802.11 \mathrm{p} / 1609$ Protocol Family in Vehicular Environments," in Proc. IEEE ITSC, 2010, pp. 1085-1090. 